\title{
A Study on Evaluation of Scrotal Lesions by Gray Scale and Colour Doppler Ultrasonography at a Tertiary Care Hospital of South India
}

\author{
Priyan Voltaire $\mathbf{R}^{1}$, Ritesh Kondeti ${ }^{2}$ \\ ${ }^{1}$ Senior Resident, ${ }^{2}$ Assocaite Professor, Department of Radiodiagnosis, Pondicherry Institute of Medical sciences, Pondichery, \\ India
}

Corresponding author: Dr.Ritesh Kondeti, Associate Professor, Department of Radiodiagnosis, Pondicherry Institute of Medical Sciences, Pondicherry, India

DOI: 10.21276/ijcmsr.2018.3.3.34

How to cite this article: Priyan Voltaire R, Ritesh Kondeti. A study on evaluation of scrotal lesions by gray scale and colour doppler ultrasonography at a tertiary care hospital of south India. International Journal of Contemporary Medicine Surgery and Radiology. 2018;3(3):C153-C157.

\section{A B S T R A C T}

Introduction: Testicular swellings are difficult to diagnose by physical examination alone as well as to differentiate whether arising from testis itself or from extra testicular elements. Ultrasonography has proved to be an exceptional gold standard tool in diagnosis of various scrotal pathologies and conditions. The present study aimed to evaluate the role of high frequency ultrasound and colour Doppler sonography as an investigating modality in distinguishing between testicular and extra testicular pathology.

Material and Methods: A one year prospective study was conducted on 100 cases after obtaining consent and approval from ethical committee. Patients of all ages and who fulfilled inclusion criteria were included and performed USG using PHILIPS HD 11 or MINDRAY DC 7 ultra sound machines in supine position. Valsalva manoeuvre was performed in cases of Varicocele to assess dynamic study of cord structures. Colour Doppler imaging was performed to investigate extra testicular vascularisation and testicular perfusion and findings noted.

Results: Median age of study group was $38.12 \pm 1.2$ years. $51 \%$ of cases were between $21-40$ years. 40 cases of inflammatory pathology, 68 cases of non inflammatory pathology and 6 normal cases were included. Acute epididymitis was the commonest inflammatory pathology (35\%) and Hydrocele common non inflammatory pathology (48.8\%). The sensitivity and specificity of clinical diagnosis was found to be 45-55\% and 90-99\% respectively whereas Ultrasound and Colour Doppler aided diagnosis had around 100\% sensitivity and specificity.

Discussion: High-resolution ultrasonography enables in clear demonstration of morphological alterations associated with acute and chronic scrotal inflammatory diseases. When colour Doppler sonography is supplemented with High frequency grey scale US, the sensitivity of diagnosing acute scrotal pathology will be increased.

Key Words: High Resolution Ultrasonography, Doppler sonography, Hydrocele, Acute epididymitis.

\section{INTRODUCTION}

Scrotum is a fibro muscular cutaneous bag containing right and left testes, the epidydimis and the lower part of the spermatic cord with associated facial coverings. These structures are affected by a wide variety of pathological conditions which may be congenital, simple inflammatory and neoplastic conditions. ${ }^{1}$ Most of the conditions of scrotum are painful and few are non-painful. Masses in the scrotum may be grouped into testicular arising from the scrotum and extra testicular arising from other structures in the scrotum. Testicular examination physically is easily accessible and consequently one should presuppose that clinical diagnosis of a scrotal swelling would be straightforward. ${ }^{2}$ Certain testicular swellings are difficult to diagnose by physical examination alone as well as to differentiate whether arising from testis itself or from extra testicular elements. The normal examination may over look significant pathology and physical signs elicited may be improperly interpreted. Hence to conclude physical examination alone lacks specificity for evaluating the structures of scrotum and testis. Hence there is an exceptional need of radiologist in for making diagnosis using imaging modalities. ${ }^{3}$

Ultrasonography has proved to be an exceptional gold standard tool in diagnosis of various scrotal pathologies and conditions. Because of its excellent spatial resolution it has $100 \%$ sensitivity in diagnosis and can differentiate a variety of conditions affecting testis, epidydimis and scrotum with similar clinical manifestations. Gray-scale Ultrasonography (US) in combination with color or power Doppler imaging is a well accepted technique for assessing scrotal lesions and testicular perfusion. Ultrasonography is exceptionally well suited to study of scrotum and its contents. Sonography is simple to perform, rapid, non-invasive, relatively inexpensive, easily reproducible, and widely available and does not involve irradiation of gonads. Sometimes emergency scrotal Doppler scan are required to diagnose testicular torsion. ${ }^{4}$

The present study aimed to evaluate the role of high frequency ultrasound and colour Doppler sonography as an 
investigating modality in distinguishing between testicular and extra testicular pathology.

\section{MATERIAL AND METHODS}

A prospective observational study was conducted for a period of one year from January 2016 to December 2106 by Department of radio diagnosis at a tertiary care hospital. The study design was approved by the institutional ethical committee and study protocol was followed as per the guidelines of the committee. The study was conducted for a period of one year and written consent was obtained from all the cases after explaining the details of the study. The demographic data, clinical history, of the cases were noted in a separate clinical record sheet and analyzed. All the cases irrespective of age referred from surgical, paediatric and urology departments with a scrotal mass or a scrotal pathological condition were included and performed ultra sonogram. Patients with extensive necrosis and gangrene involving the scrotum and perineal region, with known inguinoscrotal hernia and congenital lesions were excluded from the study.

Procedure of Scrotal Ultra sonogram: All the cases were performed USG using PHILIPS HD 11 or MINDRAY DC 7 ultra sound machines in supine position with a towel support between the thighs. $12 \mathrm{MHz}$ high-frequency lineararray transducers were used and each hemi-scrotum was scanned in transverse and longitudinal planes. Spermatic cord was studied in routine, and in patients with varicocele and testicular torsion. Valsalva manoeuvre was performed in cases of varicocele to assess dynamic study of cord structures. Testicular size can be determined by measuring the anteroposterior diameter on comparable transverse images of the left and right sides or by calculating testicular volume with the formula for an ellipsoid: $\mathrm{V}=\mathrm{L} \times \mathrm{W} \times \mathrm{H} \times 0.72$, where $\mathrm{V}=$ volume, $\mathrm{L}=$ length, $\mathrm{W}=$ width, and $\mathrm{H}=$ height. Colour Doppler imaging was performed to investigate extra testicular vascularization and testicular perfusion, with parameters optimized to display low flow velocities (low wall filter $[100 \mathrm{kHz}]$, low pulse repetition frequency [1-2 $\mathrm{Hz}$ ], and 70\%-90\% colour gain output settings). Power Doppler imaging was done in some cases of suspected testicular torsion or tumours to supplement conventional colour Doppler imaging. Pulsed-wave Doppler imaging of the intra-testicular and epididymal arteries will be included in cases of epididymo-orchitis and incomplete forms of testicular torsion to increase the diagnostic confidence.

Parameters for evaluation on usg and colour doppler:

1. Testicular dimension and size.

2. Testicular contour:-- Normal, Diffuse enlargement, Focal enlargement

3. Testicular echogenicity: Normal, Focal abnormality(Hypoechoic/Hyperechoic/Heterogenous), Diffuse abnormality (Hypoechoic/ Hyperechoic/ Heterogenous)

4. Epidydimis:

Enlargement:

a) Diffuse or

b) Focal (Head/ Body/ Tail)
Echogenicity:
a) Focal abnormality
Hyperechoic/ Heterogenous)
(Hypoechoic/
b) Diffuse abnormality
(Hypoechoic/

Hyperechoic/ Heterogenous)

5. Scrotal wall thickness.

6. Presence or absence of any collection in scrotal sac.

7. Presence or absence of any dilated veins.

8. Doppler assessment of Testes, Epidydimis and Vascular structures.

9. Presence of any anomalies in scrotum.

\section{STATISTICAL ANALYSIS}

Data were extracted into spreadsheet tables and the data collected were tabulated, analyzed and represented in the form of bar graphs and pie charts. Sensitivity and specificity of ultrasound aided diagnosis and clinical diagnosis were calculated and tabulated.

\section{RESULTS}

In the present study conducted, a total of 100 cases were enrolled who fulfilled the inclusion criteria. Age range in the study was 6-76 years with a median age of $38.12 \pm 1.2$ years. $32 \%$ of the cases were in the age group of $31-40$ years
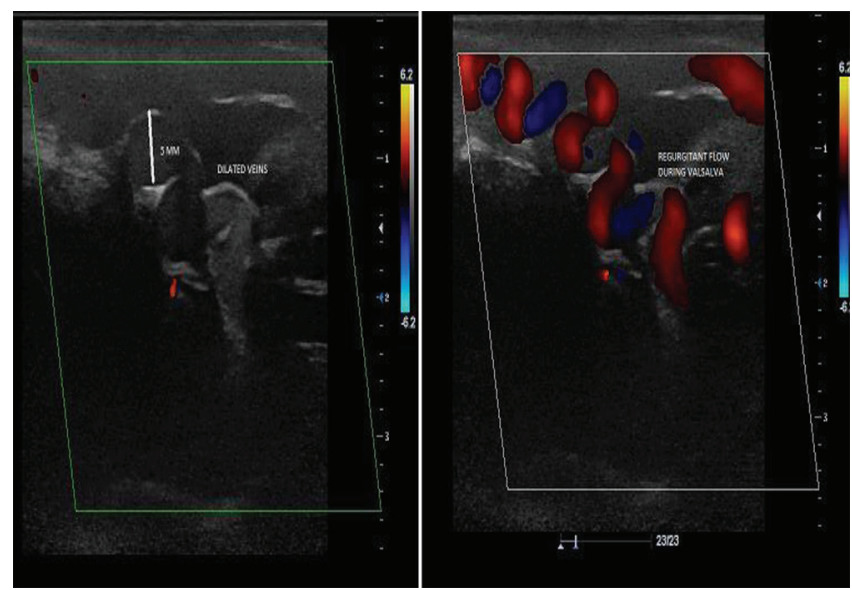

Figure-1: Colour Doppler image of Varicocele showing enlarged testicular veins in normal state and regurgitant flow on valsalva manouvere.

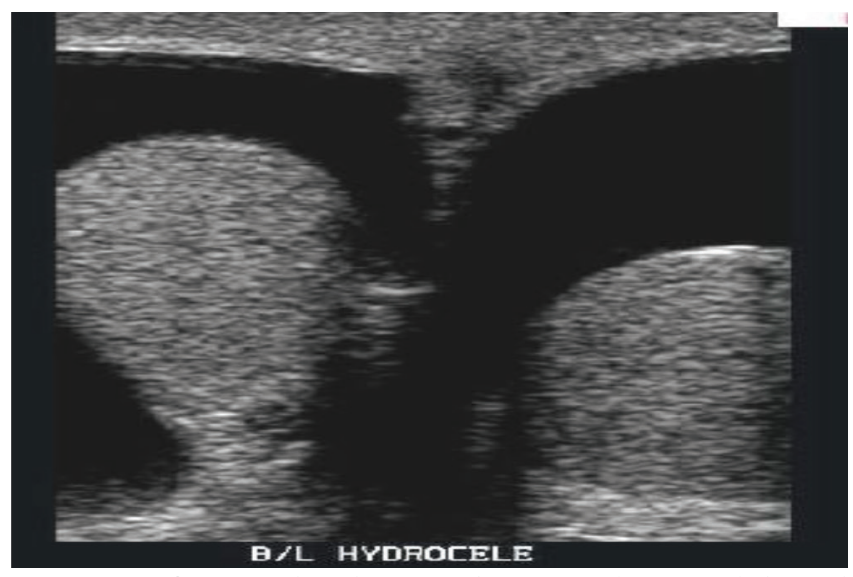

Figure-2: Gray scale ultrasound image showing bilateral extratesticular fluid collection. 


\begin{tabular}{|l|c|c|c|c|}
\hline \multirow{2}{*}{ Final Diagnosis } & \multicolumn{2}{|c|}{ Clinical Diagnosis } & \multicolumn{2}{c|}{ Ultrasound and Color Doppler Diagnosis } \\
\cline { 2 - 5 } & Sensitivity & Specificity & Sensitivity & Specificity \\
\hline Acute Epididymitis & $53.3 \%$ & $96.4 \%$ & $100 \%$ & $98 \%$ \\
\hline Acute Epididymo Orchitis & $37.5 \%$ & $86.9 \%$ & $100 \%$ & $98.9 \%$ \\
\hline Acute Orchitis & $50 \%$ & $98.9 \%$ & $67 \%$ & $100 \%$ \\
\hline Torsion & $53.8 \%$ & $96.5 \%$ & $100 \%$ & $100 \%$ \\
\hline Funiculitis & $25 \%$ & $98.9 \%$ & $80 \%$ & $100 \%$ \\
\hline Chronic Epididymo Orchitis & $33.3 \%$ & $96.9 \%$ & $100 \%$ & $100 \%$ \\
\hline Chronic Epididymitis & $20 \%$ & $98.9 \%$ & $100 \%$ & $100 \%$ \\
\hline Varicocele & $50 \%$ & $100 \%$ & $100 \%$ \\
\hline \multicolumn{2}{|r|}{ Table-1: Comparison of sensitivity and specificity of clinical diagnosis and high resolution ultrasound diagnosis } \\
\hline
\end{tabular}

\begin{tabular}{|c|c|c|c|}
\hline Final Diagnosis & $2 \times 2$ Table & Clinical Diagnosis & USG \& Color Doppler Diagnosis \\
\hline \multirow[t]{4}{*}{ Acute epididymitis } & True positive & 8 & 14 \\
\hline & False positive & 3 & 2 \\
\hline & True negative & 82 & 84 \\
\hline & False negative & 7 & 0 \\
\hline \multirow[t]{4}{*}{ Acute Epididymo orchitis } & True positive & 3 & 6 \\
\hline & False positive & 12 & 1 \\
\hline & True negative & 80 & 93 \\
\hline & False negative & 5 & 0 \\
\hline \multirow[t]{4}{*}{ Acute orchitis } & True positive & 1 & 2 \\
\hline & False positive & 1 & 0 \\
\hline & True negative & 97 & 97 \\
\hline & False negative & 1 & 1 \\
\hline \multirow[t]{4}{*}{ Torsion } & True positive & 7 & 13 \\
\hline & False positive & 3 & 0 \\
\hline & True negative & 84 & 87 \\
\hline & False negative & 6 & 0 \\
\hline \multirow[t]{4}{*}{ Funiculitis } & True positive & 1 & 4 \\
\hline & False positive & 1 & 0 \\
\hline & True negative & 95 & 95 \\
\hline & False negative & 3 & 1 \\
\hline \multirow[t]{4}{*}{ Chronic Epididymo orchitis } & True positive & 1 & 3 \\
\hline & False positive & 3 & 0 \\
\hline & True negative & 94 & 97 \\
\hline & False negative & 2 & 0 \\
\hline \multirow[t]{4}{*}{ Chronic epididymitis } & True positive & 1 & 6 \\
\hline & False positive & 1 & 0 \\
\hline & True negative & 94 & 94 \\
\hline & False negative & 4 & 0 \\
\hline \multirow[t]{4}{*}{ Varicocele } & True positive & 5 & 10 \\
\hline & False positive & 2 & 0 \\
\hline & True negative & 88 & 90 \\
\hline & False negative & 5 & 0 \\
\hline
\end{tabular}

followed in the order of 21 - 30 (19\%), 11-20 (18\%), 4150 (16\%), 0-10 (6\%), 51-60 (5\%), 61-70 and 71-80 2\% each individually. 21-40 years age group constituted a total of $51 \%$ in our study. Majority of the cases in the study presented with scrotal swelling (28\%)\%). Out of this unilateral scrotal swelling was noted in 20 cases (71.4\%), bilateral in 8 cases (28.6\%). Out of 20 unilateral scrotal swelling, 10 cases noted on left side (50\%), 10 cases on right side (50\%). Out of 28 cases, 12 cases (42.9\%) presented with acute onset, [Duration; 2 days - 10 days] 16 cases presented with insidious onset
(57.1\%) [Duration: 15 days- 6 months]. Most of the acute onset scrotal pain, particularly those associated with fever, had severe pain, whereas insidious onset not associated with fever had dull aching scrotal pain.

Inflammatory pathologies were noted in 40 cases (30.3\%), non inflammatory in 68 cases $(69.7 \%)$ and 6 cases were of normal study. Totally, pathology was noted in 132 hemiscrotum out of 100 patients studied. Acute epididymitis was the commonest inflammatory pathology observed in 14 cases (35\%) followed by acute epididymo-orchitis and chronic 
epididymitis (15\%) each individually. Other less common conditions were acute orchitis (7.5\%), chronic epididymoorchitis (7.5\%), Funiculitis (5\%), Inflammation of scrotal wall (5\%) and scrotal filariasis (2.5\%). [Figure-1]

In present study, 86 cases were detected to have non inflammatory pathology with hydrocele being the most common (48.8\%), followed by torsion testis in $15.1 \%$ cases. Other less common were Varicocele (11.7\%), Epididymal cyst ((.3\%), Trauma (9.3\%), Spermatocele (3.5\%) and neoplasm (2.3\%). [Figure-2]

On high resolution sonography, in cases of inflammatory pathologies, hyperechoic appearance was noted in 1 case of chronic Epididymo orchitis, hypoechoic appearance was observed in 14 cases of acute epididymitis, 2 cases of acute orchitis and 5 cases of acute Epididymo orchitis, Isoechoic in 1 case of Acute orchitis and acute Epididymo orchitis, Heterogenous echo pattern in 6 cases of chronic epididymitis and 2 cases of chronic Epididymo orchitis.

By Colour Doppler performance, diffuse increase in vascularity was identified in 14 cases of Acute epididymitis, 2 cases of Acute orchitis, 4 cases of Acute Epididymo orchitis, 2 cases of Chronic epididymitis and 3 cases of chronic Epididymo orchitis. Focal increase in vascularity was noted in 1 case of acute orchitis and 2 cases of acute Epididymoorchitis. Diffuse decrease in vascularity was noted in 4 cases of chronic epididymitis.

The sensitivity and specificity of clinical diagnosis was found to be $45-55 \%$ and $90-99 \%$ respectively whereas Ultrasound and Colour Doppler aided diagnosis had around $100 \%$ sensitivity and specificity in acute epididymitis, acute Epididymo-orchitis, torsion testis, varicocele, chronic epididymitis, and chronic Epididymo-orchitis. [Table-1 and 2]

\section{DISCUSSION}

The superficial location of the scrotal contents makes them ideally suited for sonographic examination. The development of high frequency, real time scanners have enhanced the diagnostic accuracy of scrotal sonographic examinations. Scrotal ultrasound has reached a level of maturity that allows the technique to be the first and only imaging examination necessary to evaluate the scrotal contents.

In the present study, $50 \%$ of the cases accounted to the age group between 21-40 years which is similar to the findings of Arger et al who mentioned that $64 \%$ of cases in his study were of age group 21-40 years. ${ }^{5}$ Scrotal swelling was the commonest clinical presentation which is similar to many studies universally. Few of the studies in Africa and eastern states reported pain, associated with mass were the commonest clinical presentation which is contrary to the findings of our study. However Willscher et al in a study of 43 patients, noted 12 cases of Inflammatory pathology and 28 cases of non inflammatory causes in his study which is similar to the findings in our study. ${ }^{6}$ Acute epididymitis was the commonest inflammatory pathology detected in our study with $35 \%$ and acute epididymo-orchitis, chronic epidydimitis accounting to 6 cases each (15\%). Similar findings were reported by Horstman et al who reported acute epididymitis present in 25 cases (49\%), acute Epididymo- orchitis in 19 cases in his study which is on par with findings of our study. ${ }^{7}$ Farriol et al, in their study of 25 cases of acute inflammatory diseases of scrotum using high-resolution grey scale and power Doppler sonographic study, found epididymitis in 11 cases (44\%), Epididymo-orchitis in 10 cases (40\%), orchitis in 2 cases (8\%), funiculitisin 2 cases $(8 \%) .^{8}$ In addition in our study, there is increased incidence of chronic Epididymo-orchitis, mainly due to large incidence of tubercular Epididymo-orchitis, the incidence of which is less in western population.

\section{High frequency US and colour Doppler appearance of inflammatory scrotal pathology}

All the 14 cases of acute epididymitis in our study exibited diffuse hypoechogenicity with diffuse increase in vascularity, and size of epididymis was increased. These findings are similar to the findings of Siddiqui $\mathrm{EH}$ et al, in their study of 25 cases. $^{9}$ In the present study, of 6 cases of acute Epididymo-orchitis, we observed diffuse hypoechogenicity in 5 cases and diffuse increase in vascularity in 4 cases, size of epididymis was increased in 6 cases. These findings are similar to the findings of Grainger et al. ${ }^{10} \mathrm{On}$ high frequency US sonography, two cases showed diffuse hypoechogenicity and 1 case was isoechoic. On colour Doppler sonography, all three cases showed increased vascularity in the areas of areas of hypoechogenicity. We noted chronic Epididymo-orchitis in 3 cases (45\%). On High-frequency US sonography, we observed diffuse increase in size of epidydimis in all 3 cases, normal size of testis in 2 cases. There was heterogenous echotexture in 2 cases, hyper echogenicity in 1 case. There was evidence of epididymal calcification seen in 1 case, testicular micro calcification in 1 case. On colour Doppler sonography, there was evidence of diffuse increase in vascularity in all 3 cases. Of 3 cases, all were proven by histopathology to be of tubercular aetiology. All the findings of our study were on par with the findings in the study of Ralls PW et al. ${ }^{11}$ In the present study, 40 cases of scrotal inflammatory pathologies, were observed with 5 cases of funiculitis, 2 cases of scrotal wall Cellulitis and 1 case of scrotal filariasis. In Cellulitis of scrotal wall, High-frequency US sonography showed loss of normal uniform hypoechoic appearance of scrotal wall, thickening of scrotal wall, presence of normal testis, epididymis and tunical sacwhich were similar to those of Luker and Siegel et al in their study. ${ }^{12}$

\section{Non inflamatory scrotal pathology}

In present study, of 86 non-inflammatory scrotal pathologies, 84 cases were non neoplastic and 2 cases of neoplastic swellings were detected sonologically. Neoplastic lesion was histo-pathologically confirmed as seminoma and 1 case exhibited well defined, homogenous hypo-echotexture and the other multiple, well defined hypo echoic areas with increased vascularity in both. In our study, of the noninflammatory pathologies observed, 63 cases were extratesticular and 23 cases were intra-testicular pathologies. The various pathologies detected in our study shows similar distribution in comparison to previous studies of Willscher et al. ${ }^{13}$ Among non-neoplastic scrotal swellings, hydrocele was the commonest pathology noted in 42 cases (48.8\%) and appeared as collection of clear fluid between two layers 
of tunica. In encysted hydrocele of cord, the collection of clear fluid along spermatic cord appeared as anechoic lesions adjacent to spermatic cord that moves with gentle traction to cord. In our study, torsion testes found in 13 cases (15.1\%), followed by varicocele in 10 cases (11.6\%). A varicocele was considered to be present by high-frequency grey scale US, if 2 or more veins could be identified, with at least 1 vein having diameter of $3 \mathrm{~mm}$. or greater. A varicocele was considered to be present by colourDoppler US, if retrograde flow was identified within the pampiniform plexus spontaneously and/ or during Valsalva manoeuvre. In this study, we studied about the positive predictive value of colour Doppler sonography in identifying varicoceles in cases of male infertility, as compared to physical examination and found that colour Doppler Ultrasonography has a positive predictive value of $100 \%$ and sensitivity of $100 \%{ }^{14}$

In our study of 86 of non-inflammatory scrotal pathologies, we noted 8 cases of epididymal cysts, 3 cases of Spermatoceles. Out of 8 cases of epididymal cysts 2 cases showed multiple cysts. Most of the epididymal cysts were uniloculated, situated in the head of epidydimis, thin walled anechoic. Associated ipsilateral hydrocele was noted in 3 cases. In present study, we detected 3 cases of spermatocele, which appeared on high frequency US scan as cystic structure with fluid level or fluid-debris level. On high frequency US scan, 2 cases of haematocele appeared as anechoic collection without any internal septations or sediments. 2 cases showed multiple septations and internal echoes within the collection. Out of the 4 cases of haematocele detected 2 were isolated finding and 1 was associated with testicular fracture and another one with testicular hematoma. In this study, we compared Sonological diagnosis with clinical diagnosis in selected common pathologies which shows physical examination has a sensitivity of (45-55\%) and specificity (90-99\%), whereas high frequency ultrasound and colour Doppler aided diagnosis had around $100 \%$ sensitivity and specificity in acute epididymitis, acute epididymo-orchitis, torsion testis, varicocele, chronic epididymitis and chronic epididymoorchitis. All the findings of our study were on par with the reports of Barton JW et al. ${ }^{15}$

To conclude High-resolution ultrasonography enables in clear demonstration of morphological alterations associated with acute and chronic scrotal inflammatory diseases, but has the limitations, because it does not enable assessment of perfusion of scrotum and its contents. When colour Doppler sonography is supplemented with High frequency grey scale US, the sensitivity of diagnosing acute scrotal pathology will be increased. In addition, Colour Doppler sonography accurately differentiates between testicular ischemia and torsion from acute inflammatory diseases in acute painful scrotal conditions. High frequency real time sonography is highly sensitive in distinguishing scrotal mass as either testicular or extra testicular masses and is clearly superior to clinical diagnosis.

High frequency ultrasonography with Doppler is highly sensitive in demonstrating the dilated, tortuous veins of pampiniform plexus and flow reversal on Valsalva maneuver. When compared to physical examination, it is highly sensitive in detecting sub clinical cases of varicoceles. High frequency ultrasonography is highly sensitive in differentiating solid from cystic scrotal masses and is highly sensitive in picking up of small, clinically impalpable intratesticular lesions like cyst, calcifications or small malignant foci. We conclude that High-frequency ultrasonography and colour Doppler sonography is an extremely valuable tool in evaluation of scrotal and testicular pathologies.

\section{REFERENCES}

1. Gutman H, Golimbu M, Subramanyam BR. Diagnostic ultrasound of scrotum. Urology 1986;27(1):72-75.

2. Vijayaraghavan SB. Sonographic differential diagnosis of acute scrotum: real-time whirlpool sign, a key sign of torsion. J Ultrasound Med.2006;25(3):563-74.

3. Middleton WD, Siegel BA, Melson GL, Yates CK, Andriole GL. Acute scrotal disorders: prospective comparison of color Doppler US and testicular scintigraphy. Radiology. 1990; 177(1):177-81.

4. Middleton WD, Thorne DA, Melson GL. Color Doppler ultrasound of the normal testis. AJR Am J Roentgenol. 1989;152(2):293-7.

5. Arger PH, Mulhern CB, Coleman BG, Pollack HM, Wein A, Koss J, et al. Prospective analysis of the value of scrotal ultrasound. Radiology. 1981;141 (3):763-6.

6. Willscher MK, Conway JF, Daly KJ, DiGiacinto TM, Patten D. Scrotal ultrasonography. J Urol. 1983;130(5):931-2.

7. Horstman WG, Melson GL, Middleton WD, Andriole GL. Testicular tumors: findings with color Doppler US. Radiology. 1992;185(3):733-7.

8. Farriol VG, Comella XP, Agromayor EG, Crèixams XS, Martinez De La Torre IB. Gray-scale and power doppler sonographic appearances of acute inflammatory diseases of the scrotum. J Clin Ultrasound JCU. 2000;28(2):67-72.

9. Siddiqui EH, Siddiqui S, Rasool G, Khan N. Scrotal pathologies; role of high resolution and Doppler ultrasound in evaluation. Professional Med J. 2013; 20(6):924-928.

10. Grainger AS, Hide IG, Elliot ST. The ultrasound appearance of scrotal odema. Eur J UItrasound 1998;8(3):33-37.

11. Ralls PW, Jensen MC, Lee KP, Mayekawa DS, Johnson MB, Halls JM. J Clin Ultrasound. 1990; 18(5):383-6.

12. Luker GD, Siegel MJ. Color Dopplersonography of the scrotum in children. AJR Am J Roentgenol. 1994;163(3):649-55.

13. Willscher MK, Conway JF, Daly KJ, DiGiacinto TM, Patten D. Scrotal ultrasonography. J Urol. 1983; 130(5):931-2.

14. R B Meacham RRT. The incidence of varicoceles in the general population when evaluated by physical examination, gray scale sonography and color Doppler sonography. J Urol. 1994; 151(6):1535-8.

15. Barton JW, Brown JM, Hammers LW, et al. Quantitative doppler assessment of acute scrotal inflammation. Radiology 1995;197(2):427-31.

\section{Source of Support: Nil; Conflict of Interest: None}

Submitted: 10-09-2018; Accepted: 17-09-2018; Published online: 26-09-2018 